\title{
A linear multisensor PHD filters via the measurement product space
}

\author{
Weifeng Liü, ${ }^{\mathrm{a}, \mathrm{b}, *}$, Yimei Chen ${ }^{\mathrm{a}}$, Chenglin Wen ${ }^{\mathrm{a}}$, Hailong Cui ${ }^{\mathrm{a}}$ \\ a School of Automation, Hangzhou Dianzi University, Xiasha Higher Education Zone, 310018 Hangzhou, P. R. China. \\ ${ }^{b}$ Science and Technology on Electro-optic Control Laboratory, Luoyang 471000, P. R. China.
}

Communicated by X. Liu

\begin{abstract}
The probability hypothesis density (PHD) is the first moment of RFS. Its integral over any region gives the expectation number of targets in that region. In the finite set statistics (FISST) framework, the PHD recursion, or PHD filter, approximate the multi-target Bayes recursion. This paper deals with the multisensor PHD filter under a linear correlation condition through multisensor product space and the measurement dimension extension (MDE) approach, which remains the similar appearance like the conventional PHD filters except the product space and some parameters in the filters. However, in the product space the dimension extended measurements may greatly increase the computational load. Therefore, we propose a fast algorithm for the linear multisensor PHD (LM-PHD) filters to increase the running speed and with cost of slightly sacrificing the tracking performance. (C)2017 All rights reserved.
\end{abstract}

Keywords: Linear correlation, random finite set, PHD filter, dimension extension of measurements, product space. 2010 MSC: 93E10.

\section{Introduction and preliminaries}

The target tracking is widely used in various fields such as military like radar tracking [5, 7, 24], public safety including pedestrian tracking $[6,35]$, medicine study $[3,8]$, space science $[2,21,27]$. The aim of target tracking is to continuously detect the measurements from the targets, and further estimate the states of targets, the number of targets and their trajectories. The target tracking can be classified into two classes: the association based approaches [1,22], which are the traditional and classic algorithms and have been studied in the past several decades. Another class is the non-association based approaches. The typical and popular non-association algorithms is the random finite set (RFS) based approaches.

A full Bayesian formulation of the multisensor-multitarget tracking problem is the Finite Set Statistics (FISST) approach proposed by Mahler $[15,17]$. Over the last decade, FISST-based approaches such as the Probability Hypothesis Density (PHD) [13, 28], Cardinalized PHD [14, 31], and multi-Bernoulli filters $[14,32]$. Since it was proposed by Mahler, the probability hypothesis density (PHD) filter has been widely studied in target tracking community. The particle-PHD filter was first given in serial references $[26,29,36]$. The particle-PHD filter can deal with the nonlinear tracking system, but it needs more

\footnotetext{
*Corresponding author

Email addresses: dashan_liu@hdu.edu.cn (Weifeng Liu), chenyimei245600@163.com (Yimei Chen), wencl@hdu.edu.cn (Chenglin Wen), 16528585@qq. com (Hailong Cui)

doi:10.22436/jnsa.010.05.12
} 
computational load. The further important work is the Gaussian mixture PHD (GM-PHD) filter due to Vo et al. [28]. The GM-PHD filter can estimate the target states without state clustering algorithms. To improve the state estimation, Nandakumaran et al. proposed the PHD smoother [18, 19, 33]. For CPHD filter, Mahler further proposed the CPHD filter [15] to reduce the estimated error of the cardinality. The CPHD filter propagates not only the PHD intensity, but also the probability distribution of target cardinality. The analytic solutions of the CPHD was proposed by Vo et al. [31]. A recent break-through in multitarget tracking is the labeled RFS based filters such as generalized labeled multi-Bernoulli (GLMB) and labeled multi-Bernoulli (LMB), which have closed form approximation solutions in the labeled RFS framework and can also output target tracks [4, 23, 30, 34].

Nevertheless, the existing RFS based algorithms are based on the single-sensor observation. Mahler investigated the multisensor PHD filter in reference [13] and pointed out that the resulting PHD formula is impractical due to its complexity in multisensor case. An approximation multisensor PHD algorithm through the convolution of individual sensor PHDs was proposed. In conference paper [16], Mahler proposed an approximate multisensor CPHD and PHD filter. [9] considered the joint estimation and registration for the PHD filter. In all these papers, the sensors are assumed to be independent.

In practice, even if we observe a single target using two sensors, the measurements are usually dependent (can be state independent). In sensor network environment, individual sensors communicate information and thus the observations coming from the sensors are also dependent. In essence, under independence of the sensors, the joint multisensor likelihood can be formulated into the product of individual sensors. Therefore, the assumption can be further relaxed.

For simplicity, in this paper we confine our problem to the linear multisensor, or sensor with linear correlation. We propose a linear multisensor PHD (LM-PHD) filter by using the dimension extended measurements approach. This approach not only effectively reduces the difficulty but also deals with sensors with linear correlation. To deal with the case of missing detection, we give an empty set to show the missing measurements and an operator of the empty set. Besides, in order to increase the computational speed, a fast algorithm is proposed.

Some preliminary results are given in reference versions [11, 12]. This paper is a more complete version. This paper is organized as follows. Section 2 and Section 3 are about background and problem description. Section 4 shows the proposed LM-PHD filter. To reduce the computational load, a fast algorithm is given in Section 5. Section 6 proposes a three-target tracking simulation. At last Section 7 concludes this paper.

\section{Background and problem description}

\subsection{Random finite sets}

In the RFS framework, the multi-target states can be described by a set as $X_{k}=\left\{x_{k, 1}, \cdots, x_{k, N(k)}\right\} \in$ $\mathcal{F}(X)$ [15], where the number of members is also random in a discrete space besides state. It can be seen as a set-valued random variable $x_{k}$ and involves all the target dynamic information. Similarly, we can model the observation as another FRS $Z_{k}=\left\{z_{k, 1}, \cdots, z_{k, M(k)}\right\} \in \mathcal{F}(Z)$, which models the clutter disturbance, missed detection and detection of targets. In the two RFSs, $x \subseteq \mathbb{R}^{n_{x}}$ and $z \subseteq \mathbb{R}^{n_{z}}$ are respectively the state space and observation space, $\mathcal{F}(X)$ and $\mathcal{F}(Z)$ are the spaces of finite subsets of $X$ and $z$, respectively. The target evolving cases such as surviving targets, new born targets and spawned targets can be formulated by a RFS union [13]:

$$
X_{k}=\left[\cup_{x \in X_{k-1}} S_{k \mid k-1}(x)\right] \cup\left[\cup_{x \in X_{k-1}} B_{k \mid k-1}(x)\right] \cup \Gamma_{k},
$$

where $S_{k \mid k-1}(x), B_{k \mid k-1}(x)$, and $\Gamma_{k}$ are respectively the RFSs of the surviving, spawned and new born targets.

More important, the probability density of a transition from $X_{k-1}$ at time $k-1$ to $X_{k}$ at time $k$ is given by $[13,15]$

$$
f_{k \mid k-1}\left(X_{k} \mid X_{k-1}\right)=\sum_{W \subset X_{k}} \pi_{T, k \mid k-1}\left(W \mid X_{k-1}\right) \pi_{\Gamma, k}\left(X_{k}-W\right),
$$


where $\pi_{T, k \mid k-1}(\cdot \mid \cdot)$ is the probability density of the surviving $\operatorname{RFS~}_{\mathrm{k}_{\mid k-1}}, \pi_{\Gamma, \mathrm{k}}(\cdot)$ is the probability density of spontaneous birth RFS $\Gamma_{k}$.

The multi-target states are observed by multisensor, which receive measurements. At time $k$, a target may be detected and produces a measurement $z_{k}$ with probability $P_{D}\left(x_{k}\right)$ or missed detection and gives empty set $\emptyset$ with probability $1-P_{D}\left(x_{k}\right)$. The received measurement set $Z_{k}$, probability of detection $P_{D}\left(x_{k}\right)$ and likelihood function $g\left(Z_{k} \mid x_{k}\right)$ involve all the information about multi-target state RFS $X_{k}$. The target measurement RFS plus clutter or false alarms RFS $K_{k}$ are expressed by:

$$
\mathrm{Z}_{\mathrm{k}}=\left[\cup_{\mathrm{x} \in \mathrm{X}_{\mathrm{k}}} \Theta_{\mathrm{k}}(\mathrm{x})\right] \cup \mathrm{K}_{\mathrm{k}} .
$$

We model a target generating measurement set $\Theta\left(x_{k}\right)$ can be described as a Bernoulli RFS

$$
\Theta\left(x_{k}\right)=\left\{\begin{array}{l}
\emptyset, \text { with probability } 1-P_{D}\left(x_{k}\right), \\
\left\{z_{k}\right\}, \text { with probability } P_{D}\left(x_{k}\right) .
\end{array}\right.
$$

Therefore, the measurement RFS $Z_{k}^{t}$ could be modeled by a multi-Bernoulli RFS under independent condition. Besides, the clutter RFS $K_{k}$ is formulated as a Poisson RFS with intensity $v_{k}(\cdot)$. Its probability distribution is given by:

$$
c_{k}\left(z_{k}\right)=v_{K, k}\left(z_{k}\right) / \int v_{k, k}\left(z_{k}\right) d z_{k}
$$

Under independent condition between target RFS $\Theta_{k}\left(X_{k}\right)$ and clutter RFS $K_{k}$, the probability density $\varphi_{k}\left(Z_{k} \mid X_{k}\right)$ is shown by [15]

$$
\varphi_{k}\left(Z_{k} \mid x_{k}\right)=\sum_{W \subseteq Z_{k}} \pi_{\Theta, k}\left(W \mid x_{k}\right) \pi_{k, k}\left(Z_{k}-W\right),
$$

where $\pi_{\Theta_{k}, k}\left(W \mid x_{k}\right), \pi_{k, k}\left(Z_{k}-W\right)$ are the probability densities of target-generated measurement RFS and clutter-generated RFS, respectively.

A target state set can be modeled as a Bernoulli RFS X, that is,

$$
\pi(X)=\left\{\begin{array}{l}
1-r, \quad X=\emptyset \\
r p(x), \quad X=\{x\} \\
0, \text { otherwise }
\end{array}\right.
$$

Further, a multi-Bernoulli RFS $X$ is defined on a fixed number of independent Bernoulli RFSs $X=\cup_{i=1}^{N} X^{(i)}$ with existence probability $r^{(i)}$, described by $\left\{\left(X^{(i)}, r^{(i)}\right)\right\}_{i=1}^{N}$. For $n$ members of RFS $\left\{x_{1}, \cdot, x_{n}\right\}$ in $X$, its probability distribution can be defined by $[15,32]$ :

$$
\pi\left(\left\{x_{1}, \cdots, x_{n}\right\}\right)=\prod_{j=1}^{N}\left(1-r^{(j)}\right) \times \sum_{1 \leqslant i_{1} \neq \cdots \neq i_{n} \leqslant N} \prod_{l=1}^{n} \frac{r^{\left(i_{l}\right)} p^{\left(i_{l}\right)}}{1-r^{\left(i_{l}\right)}},
$$

where $x_{1}, \cdots, x_{n}$ are independent.

\subsection{Review on the PHD filter}

\subsubsection{The $P H D$ filter}

The PHD is interpreted as the first order moment of RFS. Mahler proposed a PHD recursion algorithm as an approximation and alternative for the Bayesian estimation under finite sets statistics (FISST) framework. It is defined as follows

$$
\hat{\mathrm{N}}_{\mathrm{k}}=\mathrm{E}\left|\mathrm{X}_{\mathrm{k}} \cup \mathrm{S}\right|=\int_{\mathrm{S}} v_{\mathrm{k} \mid \mathrm{k}}\left(x \mid \mathrm{Z}_{1: \mathrm{k}}\right) \mathrm{d} x,
$$

where $\hat{\mathrm{N}}_{\mathrm{k}}$ is the expected number of targets which lie in the state-space $S,|$.$| is the number of elements$ in a set, and $v_{\mathrm{k} \mid \mathrm{k}}(\cdot)$ is the PHD. Assume at time $k-1$ that the PHD is $v_{\mathrm{k}-1 \mid \mathrm{k}-1}(\cdot)$. The PHD filter is 
recursively implemented through the following prediction and update steps [13]:

$$
\begin{aligned}
v_{k \mid k-1}(x) & =\gamma_{k}(x)+\int\left(P_{S}(u) f_{k \mid k-1}(x \mid u)+\beta_{k \mid k-1}(x \mid u)\right) v_{k-1}(x \mid u) d u, \\
v_{k}(x) & =\left\{1-P_{D}(x)+\sum_{z \in Z_{k}} \frac{P_{D}(x) L_{z}(x)}{\lambda c_{k}(z)+v_{k \mid k-1}\left(P_{D}(x) L_{z}\right)}\right\} v_{k \mid k-1}(x),
\end{aligned}
$$

where $\lambda$ is the average number of clutter points per scan, $c_{k}(z)$ is density of each clutter point, $\gamma_{k}(x)$ is the intensity for new born target RFS, $\beta_{k \mid k-1}(x \mid u)$ is the intensity of spawning target RFS that has the state $u$ at time $\mathrm{k}-1, \mathrm{P}_{\mathrm{S}}(\mathrm{x})$ is the target survival probability, and $\mathrm{P}_{\mathrm{D}}(\mathrm{x})$ is detection probability. By integrating of the PHD intensity, one can derive the number of targets. The peaks of the PHD distribution indicate the potential target states. The peak extracting technique is often adopted to derive the states. Under some conditions, the above PHD filter has an analytic solution of Gaussian mixture PHD filter [28].

\section{Problem formulation}

Consider the following linear multisensor multitarget system

$$
\begin{aligned}
& x_{k+1}=F_{k} x_{k}+G_{k} w_{k}, \\
& y_{k+1, j}= \begin{cases}\emptyset, & \text { with probability } 1-P_{D, j}\left(x_{k+1}\right), \\
H_{k+1, j} x_{k+1}+v_{k+1, j}, & \text { with probability } P_{D, j}\left(x_{k+1}\right), j=1,2, \cdots, s,\end{cases} \\
& z_{k+1}=A_{k+1} y_{k+1}, \\
& y_{k+1}=\left[y_{k+1,1}^{\top} \cdots, y_{k+1, s}^{\top}\right]^{\top},
\end{aligned}
$$

where $F_{k}$ is the transition matrix of the state of target, $x_{k+1}$ is the state of target, $G_{k}$ is the matrix of process noise, $w_{k}$ and $v_{k+1, j}$ are the process and $j$-th observation noise. $H_{k+1, j}$ is the observation matrix, the observation coming from the s-th sensor, the total number of sensors is $j . P_{D, j}\left(x_{k+1}\right)$ is the probability of detection for sensor $j$ at state point $x_{k+1} \cdot y_{k+1, j}$ is the local measurements, while $z_{k+1}$ denotes the extended measurements. $A_{k+1}$ is the dependent matrix among sensors. Usually, it is modeled by a serial of weighted coefficients. That is, $A_{k+1}=\left[a_{k+1}^{i, j}\right]_{n_{z} \times n_{z}}$ and

$$
a_{k+1}^{i, 1}+a_{k+1}^{i, 2}+\cdots+a_{k+1}^{i, n_{z}}=1, \quad a_{k+1}^{i, j} \in[0,1] .
$$

In above equations, we model the individual sensors by using a Bernoulli RFS. Specifically, each target is detected with probability $P_{D, j}\left(x_{k+1}\right)$ and lost with probability $1-P_{D, j}\left(x_{k+1}\right)$. We can rewrite the above system function as follows.

$$
\begin{gathered}
x_{k+1}=F_{k} x_{k}+G_{k} w_{k}, \\
z_{k+1}=H_{k+1}^{A} x_{k+1}+v_{k+1}^{A}, \\
H_{k+1}^{A}=A_{k+1} \otimes\left[H_{k+1,1}^{\top}, \cdots, H_{k+1, s}^{\top}\right]^{\top}, \\
v_{k+1}^{A}=A_{k+1} \otimes\left[v_{k+1,1}^{\top}, \cdots, v_{k+1, s}^{\top}\right]^{\top}, \\
v_{k+1}=\left[v_{k+1,1}^{\top}, \cdots, v_{k+1, s}^{\top}\right]^{\top} .
\end{gathered}
$$

It should be noted that the dimension of multisensor observation $y_{k+1}$ may be various due the missing detection of a target. For example, if a target is lost by sensor 1 at time step $k+1$, then $y_{k+1, j}$ is an empty set. Thus, the multisensor observation $y_{k+1}=\left[\emptyset, y_{k+1,2}^{\top}, \cdots, y_{k+1, s}^{\top}\right]^{\top}$. The changing dimension in the multisensor observation $y_{k+1}$ will increase the computational difficulty. In order to tackle this problem, we propose an operator named empty operator, which operates just for empty set and other elements (may empty or no empty member). Each empty set has the dimension as the original observation (under a detected case). $\otimes$ is a product operator of two matrices. We will discuss all these operators in the following section. 
Our goal is to estimate the state of target $x_{k+1}$ using the extended measurements $z_{k+1}$ under a cluttered environment. Specifically, each sensor can be seen as a single sensor space. Thus, the multisensor form a multisensor product space. Figure 1 shows that multisensor are transferred to be a product space.

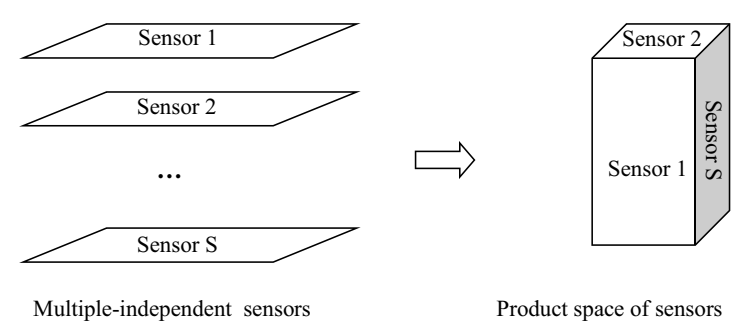

Figure 1: The space of multiple independent sensors to product space of multiple sensors.

The product space belongs to a higher dimensional space compared with a single sensor space. The measurement dimensional extension approach is used to describe the measurement product space and the correlation of the sensors.

\section{The linear multi-sensor PHD filter}

The proposed LM-PHD filter in the predicted step is the same as the original PHD filter. Hence, we focus on the update step.

\subsection{The LM-PHD update}

Suppose that multisensor RFSs are $\left\{\Sigma_{k}^{[1]}, \cdots, \Sigma_{k}^{[s]}\right\}$. In Bayesian update step, the proposed LM-PHD filter is derived by probability generating function (PGL) $F\left[g_{1}, \cdots, g_{s}, h\right]$

$$
\begin{aligned}
D_{k+1}\left(\boldsymbol{x} \mid Z^{k+1}\right) & =\frac{1}{f_{k+1}\left(Z_{k+1} \mid Z_{k}\right)} \frac{\delta^{m_{s}} F \cdots \delta \delta^{m_{1}} F \delta F}{\delta^{m_{s}} z^{\left[m_{s}\right]} \cdots \delta z^{\left[m_{1}\right]} \delta x}[\underbrace{0, \cdots, 0}_{s}, 1] \\
F\left[g_{1}, \cdots, g_{s}, h\right] & =\int \underbrace{\int \cdots \int}_{s} h^{X} g_{1}^{Z^{[1]}} \cdots g_{s}^{Z^{[s]}} f_{k+1}\left(Z^{[1]}, \cdots, Z^{[s]} \mid X\right) f_{k+1 \mid k}\left(X \mid Z_{k}\right) \delta Z^{[1]} \cdots \delta Z^{[s]} \delta X, \\
\frac{\delta^{m_{s} F}}{\delta^{m_{s}} z^{\left[m_{s}\right]}} & =\frac{\delta^{m_{s} F}}{\delta \boldsymbol{z}_{m_{s}} \cdots \delta \boldsymbol{z}_{1}}, \boldsymbol{z}_{m_{s}}, \cdots, \boldsymbol{z}_{1} \in Z^{[s]} .
\end{aligned}
$$

Obviously, it is intractable to obtain the update PHD $D_{k+1}\left(\mathbf{x} \mid Z^{k+1}\right)$. We adopt the extended measurements (3.1) of all sensors. Then all sensors are formulated by measurement product space. Nevertheless, in the product spaces, the dimension extended measurements are mutually independent. Thus above multisensor PHD reduces to the form of single-sensor PHD [13, pp: 1173, equations (110), (111)]. However, the extended measurements consist of total combination of all sensor measurements. That is

$$
D_{k+1}\left(\boldsymbol{x} \mid Z^{k+1}\right)=\frac{1}{f_{k+1}\left(Z_{k+1} \mid Z^{k}\right)} \frac{\delta^{L_{s}+1} F}{\delta z_{L_{s}}^{s} \cdots \delta z_{1}^{s} \delta x}[0,1],
$$

where $L_{s}$ is the total combination number of measurements of individual sensors. It equals to $L_{s}=$ $\prod_{l=1}^{s} m_{l}$, where $m_{l}$ is the number of measurements belonging to the lth sensor.

$$
\begin{aligned}
& \mathrm{F}_{\mathrm{k}+1}\left(\mathrm{Z}_{\mathrm{k}+1} \mid \mathbf{x}\right)=1-\mathrm{P}_{\mathrm{D}}(\mathbf{x})+
\end{aligned}
$$

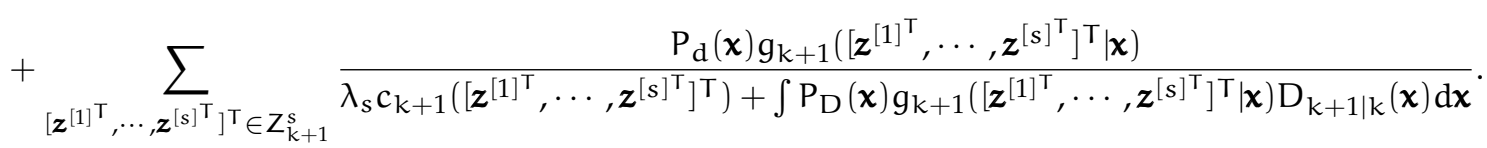


Equation (4.2) is the same as a single sensor in appearance. Nevertheless, it describes a linear multisensor system and also the correlation among the sensors. The detail proof is given in Appendix A. We thus derive the LM-PHD filter by using the approach of single-sensor PHD update. However, some parameters which are different from the single-sensor PHD filter are required to be calculated.

\subsection{The linear multi-sensor Gaussian-mixture PHD filter}

Given the following conditions

- The target movement follow linear dynamic model and multi-sensors are of linear and Gaussian.

- The survival probabilities and detection probabilities of multi-sensors are all state independent.

- The intensities of the birth and spawn RFSs are Gaussian mixtures.

- The all sensors are linear correlation.

Assume the predicted intensity is a Gaussian mixture with the following form:

$$
v_{k \mid k-1}(x)=\sum_{l=1}^{\mathrm{L}_{k \mid k-1}} \omega_{k \mid k-1}^{(\mathrm{l})} \mathcal{N}\left(x ; \mathrm{m}_{\mathrm{k} \mid \mathrm{k}-1}^{(\mathrm{l})}, \mathrm{P}_{k \mid k-1}^{(\mathrm{l})}\right) .
$$

Then, the LM-PHD intensity at time $k$ is Gaussian mixture and given by:

$$
v_{k}(x)=\prod_{j=1}^{s}\left(1-P_{D, k}^{j}\right) v_{k \mid k-1}(x)+\sum_{z \in Z_{k}} v_{D, k}(x ; z),
$$

where

$$
\begin{aligned}
& V_{D, k}(x ; z)=\sum_{l=1}^{\mathrm{L}_{k \mid k-1}} \omega_{k}^{(l)}(z) \mathcal{N}\left(x ; m_{k \mid k}^{(l)}(z), P_{k \mid k}^{(l)}\right), \\
& \omega_{k}^{(l)}(z)=\frac{P_{D, k}(z) \omega_{k \mid k-1}^{(l)} q_{k}^{(l)}(z)}{k_{k}(z)+P_{D, k}(z) \sum_{\ell=1}^{J_{k \mid k-1}} \omega_{k \mid k-1}^{(l)} q_{k}^{(l)}(z)}, \\
& m_{k \mid k}^{(l)}(z)=m_{k \mid k-1}^{(l)}+K_{k}^{(l)}\left(z-H_{k}^{A} m_{k \mid k-1}^{(l)}\right), \\
& P_{k \mid k}^{(l)}=P_{k \mid k-1}^{(l)}-K_{k}^{(l)} H_{k}^{A} P_{k \mid k-1}^{(l)}, \\
& K_{k \mid k-1}^{(l)}=P_{k \mid k-1}^{(l)} H_{k}^{A, T}\left(H_{k} P_{k \mid k-1}^{(l)} H_{k}^{A, T}+R_{k}^{A}\right)^{-1}, \\
& q_{k}^{(l)}(z)=\mathcal{N}\left(z ; H_{k}^{A} m_{k \mid k-1}^{(l)}, R_{k}^{A}+H_{k}^{A} P_{k \mid k-1} H_{k}^{A, T}\right), \\
& K_{k}(z)=\lambda c_{k}(z), \\
& P_{D, k}(z)=\prod_{j_{0} \in J_{0,}, y_{j_{0}} \in\{\emptyset\}}\left(1-P_{D, k}^{j_{0}}\right) \prod_{j_{1} \in J_{1}, y_{j_{1}} \in\{\emptyset\}} P_{D, k}^{j_{1}},
\end{aligned}
$$

in which $H^{\top}$ is the matrix transpose. As aforementioned, $z \triangleq\left[y_{1}^{\top}, \ldots, y_{s}^{\top}\right]^{\top}$, parameters $R_{k}^{A}, P_{D}(z), \lambda$ are proposed in the following subsection. $\mathrm{J}_{0}$ and $\mathrm{J}_{1}$ represent the index set of measurement of target missing and detection, respectively.

\subsection{The operator of empty set}

As aforesaid, a target may be missed detection by a sensor and thus an empty set is received from the target. Hence, we use the empty set $\emptyset$ to represent the observation. Though the empty set includes on any element, we assume it has the same dimension as an observation from the sensor. Thus the multisensor 
$z_{k+1}$ has $s \times n_{z}$ dimension, where $n_{z}$ is the dimension for each sensor. Nevertheless, an issue about the operator of empty set is imported. Hence, we define the following operator for an empty set.

$$
\begin{aligned}
\emptyset & =\emptyset \times M, \\
M & =M+\emptyset .
\end{aligned}
$$

The two operations show that the product between empty set and any number (or vector, matrix) is empty, while the addition and subtraction between empty set and any number remain the number unchanged. Therefore, (3.2) is defined by

$$
\begin{aligned}
& A_{k+1} \otimes\left[H_{k+1,1}^{\top}, \cdots, H_{k+1, s}^{\top}\right]^{\top}=\left[a h_{k+1}^{i, l}\right]_{n_{z} \times n_{x}} \\
& a h_{k+1}^{i, l}=\frac{1}{\sum_{j=1}^{n_{z}} a_{k+1}^{i, j}\left(1-\delta_{\emptyset}\left(y_{k+1, j}\right)\right)} \sum_{j=1}^{n_{z}} a_{k+1}^{i, j} h_{k+1}^{j, l}\left(1-\delta_{\emptyset}\left(y_{k+1, j}\right)\right),
\end{aligned}
$$

where $\delta_{\emptyset}\left(y_{k+1, j}\right)$ is an indicator function for empty set. If observation $y_{k+1, j}$ is not empty, then $\delta_{\emptyset}\left(y_{k+1, j}\right)=$ 0 . A weight $a_{k+1}^{i, j}$ multiplying coefficient $\left(1-\delta_{\emptyset}\left(y_{k+1, j}\right)\right)$ is used to eliminate the weights when target $j$ is missed-detection from sensor $j$ and the remaining weights are re-normalized. (3.3) is analogous.

\subsection{The parameters for the LM-PHD filters}

These parameters include clutter density $c_{k}(\cdot)$, clutter intensity $\lambda$, extension measurement covariance $\mathrm{R}_{\mathrm{k}+1}^{\mathrm{A}}$, and probability of detection $\mathrm{P}_{\mathrm{D}}^{\mathrm{s}}(\mathrm{x})$ for the product space.

\subsubsection{Clutter density and clutter intensity}

The product space is $S \triangleq S_{1} \times \cdots \times S_{s}$ and the corresponding clutter intensity is given by:

$$
\lambda=\lambda_{1} \times \cdots \times \lambda_{s}
$$

where $\lambda_{1}, \cdots, \lambda_{s}$ are the cluttered intensities of all sensors.

Under the uniform distribution of clutter measurements, the clutter density is given by:

$$
c_{k}\left(z_{k}^{c}\right)=\frac{\lambda}{V(S)},
$$

where $V(S)$ denotes the volume of the super-cylinder. The proof is given in Appendix B.

\subsubsection{The extension measurement covariance}

Assume that the process noise $w_{k}$ and measurement noise $\left\{v_{k, j}\right\}$ follow Gaussian noise with mean 0 and covariances $Q_{k}$ and $R_{k, j}$, respectively. The measurement error covariance $R_{k+1}^{A}=\operatorname{cov}\left(v_{k+1}^{A}, v_{k+1}^{A}\right)$ in (3.3) is proposed as follows:

$$
\begin{aligned}
R_{k}^{A} & =A_{k} \otimes \operatorname{diag}\left(\left[R_{k, 1}, \cdots, R_{k, s}\right]\right) \otimes A_{k}^{\top}, \\
\bar{v}_{k+1} & =E\left(A_{k+1} v_{k+1}\right)=O,
\end{aligned}
$$

where operator $\otimes$ is defined as (4.3) and (4.4), $\mathrm{O}$ is zero vector.

\subsubsection{The multi-sensor probability of detection}

Consider the probability of detection for single sensor defined by

$$
\mathrm{P}_{\mathrm{D}}(\mathrm{x})=\mathrm{P}\left(\mathrm{H}_{1} \mid \mathrm{x}, \mathrm{H}_{1}\right)=1-\mathrm{P}\left(\mathrm{H}_{0} \mid x, \mathrm{H}_{1}\right),
$$

where $\mathrm{H}_{0}$ is null hypothesis of no target existing in state point $x, \mathrm{H}_{1}$ is the alternative hypothesis, i.e., there is a target in state point $x$. In the multisensor case, if any sensor observes a target, then the target is 
considered to be detected. Therefore, define the multisensor probability of detection:

$$
P_{D}^{s}(x)=P\left(\left\{E \mid \text { At least a } H_{1}^{j}, j=1, \cdots, s\right\} \mid x, H_{1}^{1}, \cdots, H_{1}^{s}\right),
$$

where $\mathrm{H}_{1}^{1}, \cdots, \mathrm{H}_{1}^{\mathrm{s}}$ are the the alternative hypotheses for individual sensors. The event $\mathrm{E}$ shows that a target is detected by at least one sensor. We can calculate the probability using probability of misseddetection $\mathrm{P}\left(\mathrm{H}_{0} \mid x, \mathrm{H}_{1}\right)$, i.e.,

$$
\begin{aligned}
P_{D}^{s}(x) & =P\left(\left\{E \mid \text { At least a } H_{1}^{j}, j=1, \cdots, s\right\} \mid x, H_{1}^{1}, \cdots, H_{1}^{s}\right) \\
& =1-P\left(H_{0}^{1}, \cdots, H_{0}^{s} \mid x, H_{1}^{1}, \cdots, H_{1}^{s}\right) \\
& =1-\prod_{j=1}^{s} P\left(H_{0}^{j} \mid x, H_{1}^{j}\right) \\
& =1-\prod_{j=1}^{s}\left[1-P\left(H_{1}^{j} \mid x, H_{1}^{j}\right)\right]=1-\prod_{j=1}^{s}\left[1-P_{D}^{j}(x)\right] .
\end{aligned}
$$

This equation implies that the multisensor can improve the probability of detection of targets. If individual sensors have the same probability of detection $\mathrm{P}_{\mathrm{D}}(\mathrm{x})$, then (4.8) reduces to

$$
\mathrm{P}_{\mathrm{D}}^{\mathrm{s}}(\mathrm{x})=1-\left[1-\mathrm{P}_{\mathrm{D}}(\mathrm{x})\right]^{\mathrm{s}} \text {. }
$$

In the product space, the LM-PHD filter can be proceeded like the single-sensor PHD filter. Similarly, the single-sensor GM-PHD filter can also be extended to the LM-PHD filter. Despite the ability to deal with the correlation, the number of the extended measurements maybe be greatly increased. Thus, in the following section, a fast algorithm for the LM-PHD filter is considered.

\section{A fast algorithm for the LM-PHD Filter}

Since in (3.1) the measurements are dimension extension, many measurements come from clutter, instead of targets. These measurements have a less contribution to the target PHD/CPHD intensity. The measurements increase the burden of the algorithm. This motivates us to reduce the unwanted measurements as soon as possible to improve the computational speed.

But in a cluttered environment, it is difficult to remove the clutter completely to get the target measurements. In traditional association based algorithms, the validation gate is proposed to get the effective measurements. In the PHD based method many association methods have been used [10, 20] to form target tracks. We adopt the validation gate around each prediction state to derive the target measurements and reduce the effects of clutter. Nevertheless, it does not need to calculate association probabilities like the traditional association based methods.

For simplicity, a circle gate with a fixed radius is proposed in this paper. The center of the circles are w.r.t the predicted positions of the new birth targets and the surviving targets. Thus a set of effective measurements of targets can be derived.

The likelihood function $g(z \mid x)$ can be as an index to measure the dependence of a measurement $z$ to target state $x$. Therefore, we adopted a preprocessing step to reduce the number of measurements in the LM-PHD algorithm. Nevertheless, the average clutter intensity in the LM-PHD is required to be estimated. Because the Poisson distribution has an additive property, we therefore propose the following equation to derive the reduced intensity

$$
\lambda_{\text {fast }}=\frac{m_{k, \text { reduced }}}{m_{k, 1} \times \cdots \times m_{k, s}} \lambda,
$$

where $m_{k, j}$ is the number of measurements for the $j$-th sensor. The reduced measurements can be obtained by

$$
\mathrm{m}_{\mathrm{k}, \text { reduced }}=\sum_{z \in Z_{\mathrm{k}}} \Delta\left(\frac{1}{\mathrm{C}} \mathrm{g}(z \mid x)>\gamma_{z}\right)
$$




$$
C=\sum_{z \in Z_{k}} g(z \mid x)
$$

where $\gamma_{z}$ is a threshold for the normalized likelihood. The delta function $\Delta(\cdot>\cdot)$ is a logic operation and defined by

$$
\Delta(1)=1, \quad \text { or } \quad \Delta(0)=0 .
$$

The average intensity $\lambda_{\text {fast }}$ is proposed in (5.1). By this process, the number of the extended measurements are greatly reduced.

\section{Simulation results}

Consider six targets move in $x-y$ plane and the surveillance region is $[-1000,1000] \times[-1000,1000] \mathrm{m}^{2}$. The target state vector $x_{k}=\left[p_{x, k}, \dot{p}_{x, k}, p_{y, k}, \dot{p}_{y, k}\right]^{\top}$ consists of the position $\left(p_{x, k}, p_{y, k}\right)$ and velocity $\left(\dot{p}_{x, k}, \dot{p}_{y, k}\right)$. The intensity of target spontaneous birth is

$$
\mathrm{D}_{\gamma, k}(x)=0.1 \mathcal{N}\left(x ; \mu_{1,0}, \Sigma_{1,0}\right)+0.1 \mathcal{N}\left(x ; \mu_{2,0}, \Sigma_{2,0}\right),
$$

where $\mu_{1,0}=[-1000,30,1000,-30]^{\top}, \mu_{2,0}=[-1000,30,-1000,30]^{\top}, \Sigma_{1,0}=\operatorname{diag}([100,25,100,25])$. The average clutter density is $\lambda=2.0 \times 10^{-5}, 80$ clutter points in the surveillance region in average. The probabilities of detection are all $\mathrm{P}_{\mathrm{s}, \mathrm{D}}=0.9$ for sensors. The process noise covariance and measurement noise covariance are $Q_{k}=\operatorname{diag}([9,9]) \mathrm{m}^{2}$. The measurement covariances are respectively $R_{k, 1}=$ $\operatorname{diag}([25,25]) \mathrm{m}^{2}, \mathrm{R}_{\mathrm{k}, 2}=\operatorname{diag}([50,50]) \mathrm{m}^{2}$ for sensors 1 and 2 . That is, the sensor 1 has a better performance than sensor 2. The reduced threshold in (5.2) is $\gamma_{z}=1.0 \times 10^{-6}$. The matrix of positional observation and dependent matrices are given as follows:

$$
\mathbf{H}_{\mathrm{k}}=\left[\begin{array}{llll}
1 & 0 & 0 & 0 \\
0 & 0 & 1 & 0
\end{array}\right], \quad \mathbf{A}_{\mathrm{k}}=\left[\begin{array}{cccc}
0.8 & 0 & 0.2 & 0 \\
0 & 0.8 & 0 & 0.2 \\
0.6 & 0 & 0.4 & 0 \\
0 & 0.6 & 0 & 0.4
\end{array}\right]
$$

\subsection{The tracking processing}

Figure 2 shows the trajectories in $x-y$ plane. We optionally adopt an estimation result. Figure 3 is the corresponding $x$ and $y$ coordinates which suggests the target start time and the end time in total 60 times. In order to test tracking performance, we implement trial through 1000 Monte Carlo runs. The final lost rate is around $10 \%$, the average implement time is $1.2 \mathrm{~s}$ for once 60 -step run. Besides, we adopt the OSPA metrics [25] to verify tracking precision.

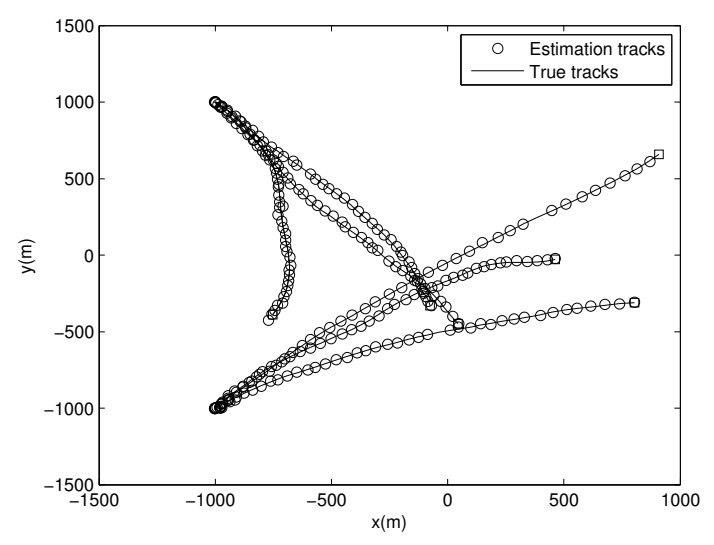

Figure 2: The target trajectories shown in xy plane, where o and $\square$ are the start and end points. 

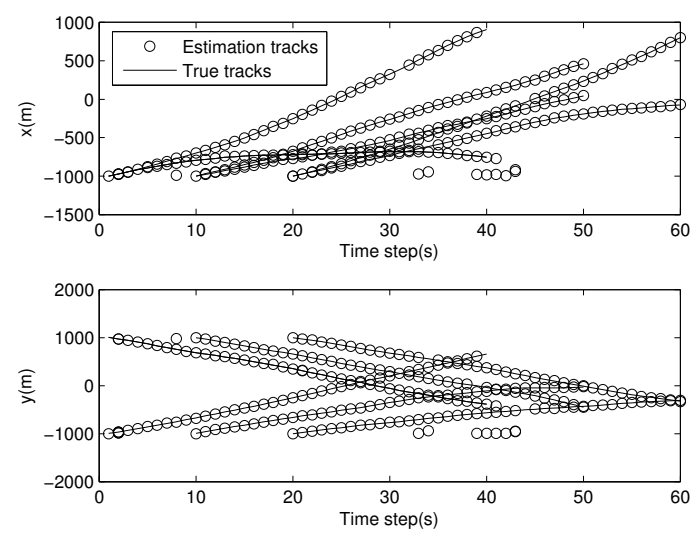

Figure 3: The target trajectories shown in $x$ and $y$ coordinates against time.

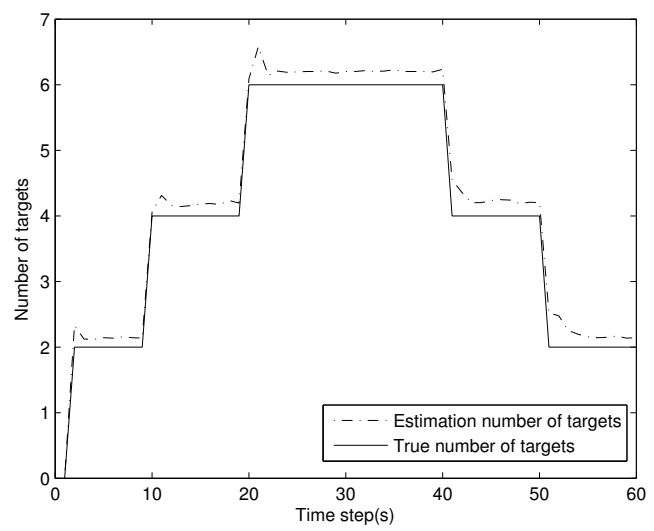

Figure 4: The number of targets against time (1000MCs).

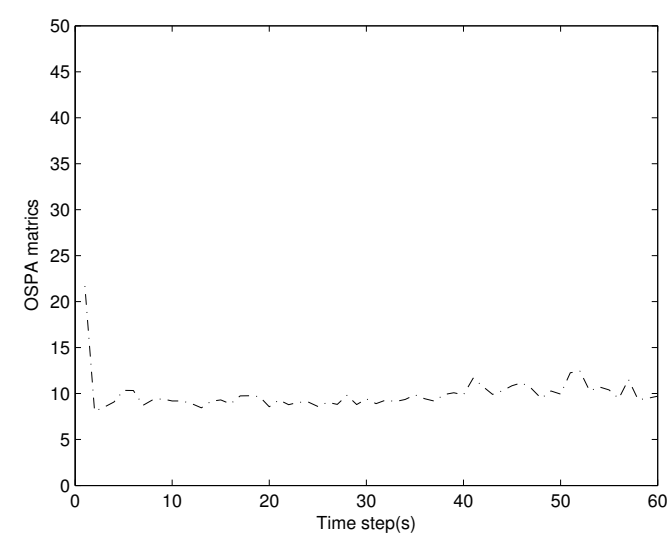

Figure 5: The OSPA matric against time (1000MCs).

\subsection{Performance comparison}

The proposed fast algorithm can greatly increase the computational speed. In the extension dimensional PHD filter, this can be seen from Figure 6 even in a relative low clutter density $\lambda=1.0 \times 10^{-5} \mathrm{~m}^{-2}$ (40 clutter density in average), the CPU computing time is $92.1 \mathrm{~s}$ for the whole 60 steps. When the clutter density reaches $4.0 \times 10^{-5} \mathrm{~m}^{-2}$, the CPU computing time increase to 1272 . In comparison, the CPU time for the fast algorithm are $1.66 \mathrm{~s}$ and $12.66 \mathrm{~s}$, respectively. 


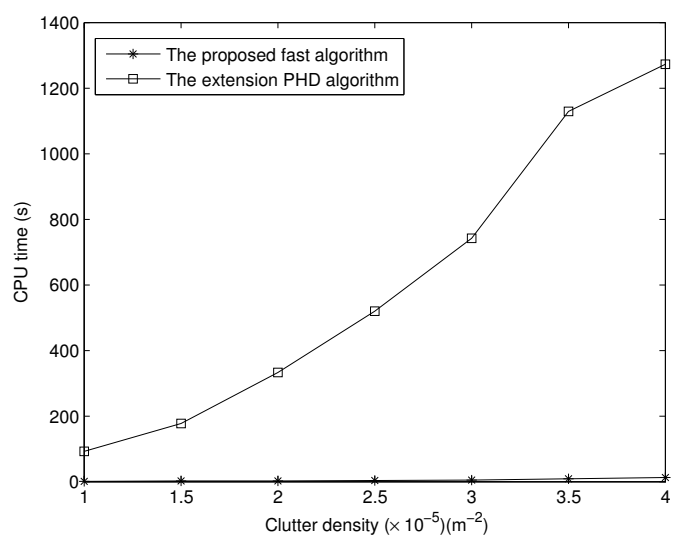

Figure 6: The computational time comparison under the different clutter density.

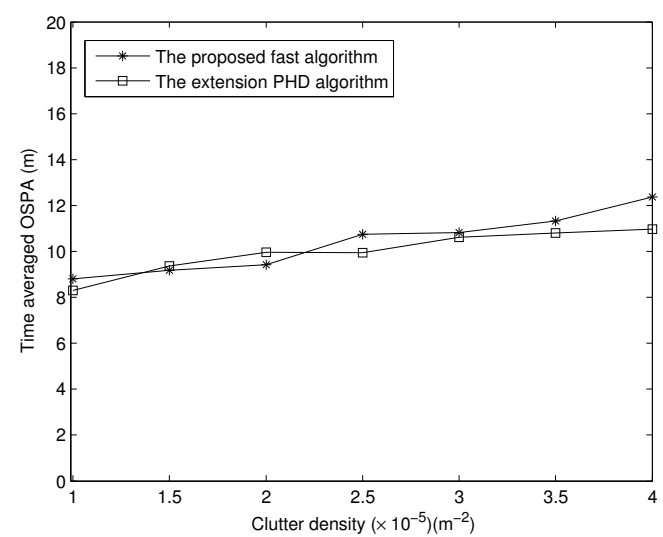

Figure 7: The OSPA comparison vs. various clutter densities.

The computational precision can be seen from Figure 7, where we give the time averaged OSPA against the clutter density. It shows that the precision has been slightly sacrificed compared with the extension PHD filter and we think it is acceptable with a view to increase computational speed.

\section{Conclusion}

In this paper, we propose a measurement product space which consists of multiple sensors. In this space, we describe the linear correlation between the sensors. Further, a LM-PHD filter is given by using the measurement dimension extension. Although the proposed LM-PHD filter has the similar form as the original PHD filter, it can deal with the case where the sensors are linear correlation. Moreover, some parameters in the LM-PHD filter are investigated. Besides, in order to reduce the computational load, a fast LM-PHD filter is proposed to accelerate the computational speed.

\section{Appendix A.}

Proof of the linear multisensor PHD filter Equations (4.1), (4.2).

In this appendix, we will show that the measurement dimension extension (MDE) PHD filter under the linear Gaussian (LG) correlation conditions of all the sensors. Our proof consists of two steps: The first is to derive the distribution function for the dimension extension vector. The second step gives the LM-PHD filter by using the Mahler's original approach. 
Let $y_{k+1}^{[l]}$ be the l-the sensor measurement vector and it follows the following Gaussian distribution

$$
f\left(y_{k+1}^{[l]} \mid x_{k+1}\right)=N\left(0, R_{k+1}^{l}\right), \quad l=1, \cdots, s .
$$

Let $z_{k+1}^{s}$ be MDE vector $z_{k+1}^{s} \triangleq\left[y_{k+1}^{[1]^{\top}}, \cdots, y_{k+1}^{[s]^{\top}}\right]^{\top}$, then the MDE vector has the following Gaussian distribution

$$
f\left(z_{k+1}^{s} \mid x\right)=N\left(O, R_{k+1}^{A}\right),
$$

where $\mathrm{O}$ is zeros matrix, $\mathrm{R}_{\mathrm{k}+1}^{\mathrm{A}}$ is given by (4.7). For a non-zero mean vector, zeros matrix will be nonzero diagonal matrix. Assume that the number of measurements for individual sensors are respectively $m_{k, 1}, \cdots, m_{k, s}$, then in the measurement product space $S$, the total number of the dimension extended measurement set $Z_{k+1}^{s}$ is $L_{s}=m_{k, 1} \times \cdots \times m_{k, s}$.

With the MDE distribution, we next show the LM-PHD filter for the update step. Given the prediction PHD is approximately Poisson

$$
\begin{aligned}
f_{k+1 \mid k}\left(X \mid z^{k}\right) & =e^{-M} D\left(x_{1}\right) \cdots D\left(x_{m}\right) \\
& =e^{-M} M^{m} s\left(x_{1}\right) \cdots s\left(x_{m}\right),
\end{aligned}
$$

where $M=\int D(x) d x, D\left(x_{i}\right)=M s\left(x_{i}\right), i=1, \cdots, m, z^{k} \triangleq\left\{z_{1}, \cdots, z_{k}\right\}, s\left(x_{i}\right)$ is the state distribution of the $i$-th state $x_{i}$. We consider the measurement dimension space $S \triangleq S_{1} \times \cdots \times S_{s}$. The linear multisensor likelihood function is $f\left(z_{k+1}^{s} \mid X\right)$, then the posterior distribution can be expressed as to be

$$
f\left(X \mid z^{k+1}\right)=\frac{1}{K} f\left(z_{k+1} \mid X\right) f\left(X \mid z^{k}\right),
$$

where $z_{k+1}$ is the measurement vector after dimension extension at time step $k+1 . k=f\left(z_{k+1}^{s} \mid z^{k}\right)$ is a normalizing constant. With this, the PGL of the posterior distribution is proposed as follows

$$
\begin{aligned}
F[g, h] & =\iint h^{X} g^{z^{s}} f\left(z_{k+1}^{s} \mid X\right) f\left(X \mid z^{k}\right) \delta X \delta z_{k+1}^{s} \\
& =\iint h^{X} g^{z_{k+1}^{s}} f\left(z^{s} \mid X\right) e^{-M} D\left(x_{1}\right) \cdots D\left(x_{m}\right) \delta X \delta z_{k+1}^{s} \\
& =\int h^{X} G_{k+1}(g \mid X) e^{-M} D\left(x_{1}\right) \cdots D\left(x_{m}\right) \delta X
\end{aligned}
$$

where $G_{k+1}(g \mid X)=\int g\left(z_{k+1}^{s} \mid X\right) \delta z_{k+1}^{s}$. With this PGL, using the following equations and Theorem 2 in reference [13] ([13, pp.1163 Equation (66)])

$$
\begin{aligned}
& f(X)=\frac{\delta^{n} \beta_{\Xi}}{\delta x_{1} \cdots \delta x_{m}}(\phi)=\frac{\delta^{n} F}{\delta x_{1} \cdots \delta x_{m}}[0], \\
& D(x)=\frac{\delta F}{\delta x}[1] .
\end{aligned}
$$

The PHD of the posterior distribution can be gotten by the PGL F $[\mathrm{g}, \mathrm{h}]$

$$
\mathrm{D}\left(x \mid z^{\mathrm{k}+1}\right)=\frac{1}{\mathrm{f}\left(z_{\mathrm{k}+1} \mid Z^{\mathrm{k}}\right)} \frac{\delta^{\mathrm{n}} \mathrm{F}}{\delta z_{\mathrm{n}} \cdots \delta z_{1} \delta x}[0,1] .
$$

In the measurement product space, the original measurement set $\left\{z_{n}, \cdots, z_{1}\right\}$ is substituted by the new MDE set $\left\{z_{\mathrm{k}+1, \mathrm{~L}_{s}}^{\mathrm{s}}, \cdots, z_{\mathrm{k}+1,1}^{\mathrm{s}}\right\}$, so the above equation is given by

$$
D_{k+1}\left(x \mid z^{k+1}\right)=\frac{1}{f_{k+1}\left(z_{k+1}^{s} \mid z_{k}\right)} \underbrace{\frac{\delta^{L_{s}+1} F}{\delta z_{k+1, L_{s}}^{s} \cdots \delta z_{k+1,1}^{s}} \delta x}_{L_{s}}[0,1],
$$


where $L_{s}$ is the total number of the measurement elements in extended measurement set $z_{k+1}^{s}$. Given

$$
\mathrm{f}_{\mathrm{k}+1}\left(z_{\mathrm{k}+1}^{\mathrm{s}} \mid z_{\mathrm{k}}\right)=\underbrace{\frac{\delta^{\mathrm{L}_{s}+1} \mathrm{~F}}{\delta z_{\mathrm{k}, \mathrm{L}_{s}}^{\mathrm{s}} \cdots \delta z_{\mathrm{k}+1,1}^{\mathrm{s}}}}_{\mathrm{L}_{s}}[0,1] .
$$

As aforesaid, in the measurement product space, the dimension extended measurements are mutually independent. Thus, we can apply Equations (116) and (112) in reference [13] ([13, pp.1174 and pp.1175]) to above (A.1). In fact, the MDE based PHD update filter has the same form with the original PHD filter except the different measurement sets. Specifically, the original PHD filter is $\left\{z_{\mathrm{k}+1,1}, \cdots, z_{\mathrm{k}+1, \mathrm{n}}\right\} \triangleq Z_{\mathrm{k}+1}$, while the MDE PHD filter is $\left\{z_{\mathrm{k}+1,1}^{\mathrm{s}}, \cdots, z_{\mathrm{k}+1, \mathrm{~L}_{s}}^{\mathrm{s}}\right\} \triangleq \mathrm{Z}_{\mathrm{k}+1}^{\mathrm{s}}$. The following proof is the same as the original PHD filter (see reference [24, pp:1173]), so we omitted it here.

\section{Appendix B.}

Proof of clutter intensity in the super-cylinder (4.5), (4.6).

Assume that in a set of sensor plane the measurement intensities follow Poisson distributions with intensities $\lambda_{1}, \cdots, \lambda_{s}$, respectively. The measurement planes $S_{1} \times \cdots \times S_{s}$ form a spatial point processes. From the point of random Poisson point process view, the intensity of measurement in the super-cylinder equals to first order of the Poisson point process, that is

$$
\mathrm{m}_{\Sigma, 1}(z)=\mathrm{E}\left[\delta_{\Sigma}(z)\right]=\int_{S} \delta_{\Sigma}(z) f(z) \mathrm{d} \mu(z),
$$

where $d \mu(z)$ is the number of Poisson point occurring inside region dS. Obviously, it is

$$
\delta_{\Sigma} f(z) d \mu(z)=\frac{\lambda_{1}}{S_{1}} \cdots \frac{\lambda_{s}}{S_{s}} d z_{1} \cdots d z_{s} .
$$

Therefore, the intensity of measurement in the super-cylinder is

$$
\begin{aligned}
\lambda & =M_{\Sigma, 1}(S)=E[|\Sigma \cap S|] \\
& =\int|\Sigma \cap S| f(z) d \mu(z) \\
& =\int|\Sigma \cap S| f(z) d \mu(z) \\
& =\int_{S} \frac{\lambda_{1}}{S_{1}} \cdots \frac{\lambda_{s}}{S_{S}} \mathrm{~d} z_{1} \cdots d z_{s} \\
& =V(S) \times \frac{\lambda_{1}}{S_{1}} \cdots \frac{\lambda_{s}}{S_{S}}=\lambda_{1} \cdots \lambda_{s} .
\end{aligned}
$$

This completes the proof.

According to the Poisson distribution, its intensity $\lambda$ can be determined by its density, for a uniform distribution of measurements in the super-cylinder $V(S)$, the clutter density is given by

$$
c_{k}\left(\left[z^{[1]}, \cdots, z^{[s]}\right]^{\top}\right)=\frac{\lambda}{V(S)} .
$$

\section{Acknowledgment}

This work was supported in part by the NSFC (61333011, 61175030, 61271144), ZJNSF (LY15F030020, U1509203) and the Aeronautical Science Foundation of China (201551T5001), Hangzhou Dianzi University (HD1232234). 


\section{References}

[1] Y. Bar-Shalom, Tracking methods in a multitarget environment, IEEE Trans. Autom. Control, 23 (1978), 618-626. 1

[2] K. Chang, M. E. Pollock, M. K. Skrehot, Space-based millimeter wave debris tracking radar, Proc. SPIE, Monolithic Microwave Integrated Circuits for Sensors, Radar, and Communications Systems, Orlando, FL, 1475 (1991), 257266. 1

[3] L. Chen, Z.-Y. Zhao, H. Yan, A Probabilistic Relaxation Labeling (PRL) based method for C. elegans cell tracking in microscopic image sequences, IEEE J. Sel. Topics Signal Process, 10 (2016), 185-192. 1

[4] H. Deusch, S. Reuter, K. Dietmayer, The labeled multi-Bernoulli SLAM filter, IEEE Trans. Signal Process., 22 (2015), 1561-1565. 1

[5] W. J. Farrell, Interacting multiple model filter for tactical ballistic missile tracking, IEEE Trans. Aerosp. Electron. Syst., 44 (2008), 418-426. 1

[6] C. Huang, Y. Li, R. Nevatia, Multiple target tracking by learning-based hierarchical association of detection responses, IEEE Trans. Pattern Anal. Mach. Intell., 35 (2013), 898-910. 1

[7] J.-W. Kim, P. Menon, E. Ohlmeyer, Motion models for use with the Maneuvering Ballistic Missile tracking estimators, Proceedings of the AIAA guidance, navigation, and control conference, Toronto, Ontario, Canada, (2010), 1-13. 1

[8] M. F. Kircher, S. S. Gambhir, J. Grimm, Noninvasive cell-tracking methods, Nat. Rev. Clin. Oncol., 8 (2011), $677-688$. 1

[9] F. Lian, C.-Z. Han, W.-F. Liu, H. Chen, Joint spatial registration and multi-target tracking using an extended probability hypothesis density filter, IET radar, sonar \& navigation, 5 (2011), 441-448. 1

[10] L. Lin, Y. Bar-Shalom, T. Kirubarajan, Track labeling and PHD filter for multitarget tracking, IEEE Trans. Aerosp. Electron. Syst., 42 (2006), 778-795. 5

[11] W.-F. Liu, C.-L. Wen, A linear multisensor PHD filter using the measurement dimension extension approach, Advances in Swarm Intelligence: the 2th International Conference on Swarm Intelligence, Chongqing, China, (2011), 486-493. 1

[12] W.-F. Liu , C.-L. Wen, The fast linear multisensor RFS-multitarget tracking filters, Proceedings of the 17th International Conference on Information Fusion, Slamanca, Spanish, (2014), 1-8. 1

[13] R. P. S. Mahler, Multitarget Bayes filtering via first-order multitarget moments, IEEE Trans. Aerosp. Electron. Syst, 39 (2003), 1152-1178. 1, 2.1, 2.2.1, 4.1, Appendix A, Appendix A

[14] R. P. S. Mahler, PHD filters of higher order in target number, IEEE Trans. Aerosp. Electron. Syst., 43 (2007), $1523-1543$. 1

[15] R. P. S. Mahler, Statistical multisource-multitarget information fusion, Artech House, Inc., Norwood, MA, USA, (2007). $1,2.1,2.1$

[16] R. P. S. Mahler, Approximate multisensor CPHD and PHD filters, Proceedings of the 13th International Conference on Information Fusion, (2010), 1152-1178. 1

[17] R. P. S. Mahler, Advances in statistical multisource-multitarget information fusion, Artech House, Inc., Norwood, MA, USA, (2014). 1

[18] R. P. S. Mahler, B.-T. Vo, B.-N. Vo, Forward-backward probability hypothesis density smoothing, IEEE Trans. Aerosp. Electron. Syst., 48 (2012), 707-728. 1

[19] N. Nandakumaran, K. Punithakumar, T. Kirubarajan, Improved multi-target tracking using probability hypothesis density smoothing, Proceedings of the SPIE Conference on Signal and Processing of Small Targets, 6699 (2007), 1-6. 1

[20] K. Panta, B.-N. Vo, S. Singh, Novel data association schemes for the probability hypothesis density filter, IEEE Trans. Aerosp. Electron. Syst., 43 (2007), 556-570. 5

[21] J. N. Pelton, Tracking of orbital debris and avoidance of satellite collisions, Handbook of Satellite Applications, Springer, New York, (2016), 1-13. 1

[22] D. B. Reid, An algorithm for tracking multiple targets, IEEE Trans. Autom. Control, 24 (1979), 843-854. 1

[23] S. Reuter, B.-T. Vo, B.-N. Vo, K. Dietmayer, The labeled multi-Bernoulli filter, IEEE Trans. Signal Process., 62 (2014), 3246-3260. 1

[24] B. G. Saulson, K.-C. Chang, Nonlinear estimation comparison for ballistic missile tracking, Opt. Eng., 43 (2004), 14241438. 1, Appendix A

[25] D. Schuhmacher, B.-T. Vo, B.-N. Vo, A consistent metric for performance evaluation of multi-object filters, IEEE Trans. Signal Process., 56 (2008), 3447-3457. 6.1

[26] H. Sidenbladh, Multi-target particle filtering for the probability hypothesis density, ArXiv, 2003 (2003), 7 pages. 1

[27] R. Suwantong, Development of the Moving HorizonEstimator with Pre-Estimation (MHE-PE), Application to Space Debris Tracking during the Re-Entries, Automatic, Supélec, (2014). 1

[28] B.-N. Vo, W.-K. Ma, The Gaussian mixture probability hypothesis density filter, IEEE Trans. Signal Process., 54 (2006), 4091-4104. 1, 2.2.1

[29] B.-N. Vo, S. Singh, A. Doucet, Sequential Monte Carlo implementation of the PHD filter for multi-target tracking, PProceedings of the International Conference on Information Fusion, Cairns, Australia, (2003), 792-799. 1

[30] B.-T. Vo, B.-N. Vo, Labeled random finite sets and multi-object conjugate priors, IEEE Trans. Signal Process., 13 (2013), 3460-3475. 1 
[31] B.-T. Vo, B.-N. Vo, A. Cantoni, Analytic implementations of the cardinalized probability hypothesis density filter, IEEE Trans. Signal Process., 55 (2007), 3553-3567. 1

[32] B.-T. Vo, B.-N. Vo, A. Cantoni, The cardinality balanced multi-target multi-Bernoulli filter and its implementations, IEEE Trans. Signal Process., 57 (2009), 409-423. 1, 2.1

[33] B.-N. Vo, B.-T. Vo, R. P. S. Mahler, Closed-form solutions to forwardbackward smoothing, IEEE Trans. Signal Process., 60 (2012), 2-17. 1

[34] B.-N. Vo, B.-T. Vo, D. Phung, Labeled random finite sets and the Bayes multi-target tracking filter, IEEE Trans. Signal Process., 62 (2014), 6554-6567. 1

[35] Q. Yu, G. Medioni, Multiple-target tracking by spatiotemporal monte carlo markov chain data association, IEEE Trans. Pattern Anal. Mach. Intell., 31 (2009), 2196-2210. 1

[36] T. Zajic, R. Mahler, Particle-systems implementation of the PHD multitarget-tracking filter, Proc. SPIE, Signal Processing, Sensor Fusion, and Target Recognition XII, 5096 (2003), 291-299. 1 